\title{
Effects of amiodarone on thyroid function in patients with ischaemic heart disease
}

\author{
David A. Pritchard, ${ }^{1}$ Bramah N. Singh, and Peter J. Hurley \\ From the Department of Cardiology, Green Lane Hospital; Department of Nuclear Medicine, Auckland Hos- \\ pital; and Department of Medicine, Auckland University School of Medicine, Auckland, Nerw Zealand
}

Thyroid function was evaluated clinically and biochemically in 12 patients with ischaemic heart disease receiving $200 \mathrm{mg}$ oral amiodarone three times daily for periods up to 6 weeks. During drug administration, no patient developed clinical or laboratory evidence of hypothyroidism, but serum levels of $T_{3}$ tended to fall and those of $T_{4}$ increased but not to levels outside the normal range. Amiodarone produced a significant reduction in heart rate with prolongation of the QTc interval of the electrocardiogram without altering either the PR interval or the $Q R S$ duration. These effects of the drug were still present 4 weeks after cessation of treatment. In spite of the high iodine content, amiodarone does not, therefore, depress thyroid function to any important degree during chronic administration and its antianginal action does not appear to be caused by the production of generalized hypothyroidism.

Amiodarone, ${ }^{2}$ a benzfuran derivative which shares structural similarities with thyroxine (Fig. I), has been used recently in the treatment of angina (Vastesaeger, Gillot, and Rasson, 1967; Barzin and Fréson, 1969) and cardiac arrhythmias (Van Schepdael and Solvay, 1970). It produces bradycardia, coronary dilatation, and a depression of myocardial oxygen consumption, without a significant effect on cardiac output (Charlier et al., I968). The drug also antagonizes the effects of sympathetic nerve stimulation, but unlike propranolol it does not produce competitive beta-adrenergic receptor blockade (Charlier, 1970). Amiodarone is devoid of local anaesthetic properties and the antiarrhythmic effects of the compound, well defined in experimental animals (Singh and Vaughan Williams, 1970) as well as man (Van Schepdael and Solvay, 1970; Rosenbaum et al., 1974), have been explained on the basis of its electrophysiological actions. Singh and Vaughan Williams (1970) showed that chronic administration of amiodarone in rabbits produced a significant increase in the duration of the action potential without an effect on the resting membrane potential or the maximal rate of de-

Received 28 February 1975.

${ }^{1}$ Present address: Cardiology Division, Mayo Clinic and Foundation, Rochester, Minnesota, U.S.A.

${ }^{2}$ Amiodarone is supplied by Services de Recherche Labaz, Brussels. It is not available for clinical use in the United Kingdom. polarization of the action potential. The overall change was similar to that seen in hypothyroidism (Freedberg, Papp, and Vaughan Williams, 1970), and it was significant that the effects on the action potential could be prevented by the simultaneous administration of thyroxine (Singh and Vaughan Williams, 1970).

Benziodarone, an earlier benzfuran derivative, was withdrawn from clinical use because it produced hypothyroidism (Harrison and Cameron, 1965). Whether or not amiodarone might also induce generalized hypothyroidism of clinical significance has not been convincingly established. The present study was, therefore, undertaken to evaluate the effects of the drug on thyroid function in patients with ischaemic heart disease.

\section{Patients and methods}

Twelve patients with ischaemic heart disease were selected from the Cardiology Department and Coronary Care Follow-up Clinic of Green Lane Hospital. The majority of the patients had proven myocardial infarction 6 to 8 weeks before entry into the study. Patients who were on digoxin therapy or beta-adrenergic blocking drugs were excluded. Before amiodarone was given, each patient was interviewed and examined for evidence of thyroid dysfunction; particular attention was paid to heart rate and blood pressure. Informed consent was obtained from all patients before the administration of amiodarone. A 12-lead electrocardiogram at a paper speed of $25 \mathrm{~mm} / \mathrm{s}$ was obtained in addition to a $5 \mathrm{~s}$ 
tracing of lead II which was recorded at a paper speed of $50 \mathrm{~mm} / \mathrm{s}$ to allow an accurate measurement of the QT interval. The QTc interval was calculated from the measured QT interval by correction for rate using the Bazett formula (Bazett, 1920).

Blood was withdrawn for the estimation of serum protein bound iodine (PBI), total serum thyroxine $\left(\mathrm{T}_{4}\right)$, total tri-iodothyronine $\left(\mathrm{T}_{3}\right)$, resin uptake of tri-iodothyronine $\left(\mathrm{T}_{3}\right.$ resin uptake), thyroid stimulating hormone (TSH), and serum cholesterol.

PBI was measured in an automatic spectrophotometer. Total $T_{4}$ and $T_{3}$ resin uptakes were measured by competitive protein binding techniques and total $T_{3}$ and TSH were measured by radio-immunoassay. Ankle jerk half relaxation time was measured with an automatic photomyograph.

When all baseline data were obtained, amiodarone was started in a dose of $200 \mathrm{mg} 3$ times daily and continued for 6 weeks. After 3- and 6-week periods of treatment with the drug, heart rate, blood pressure, QTc interval on the electrocardiogram as well as thyroid function were assessed. The drug was then discontinued and all measurements repeated 4 weeks later.

\section{Results}

Of the 12 patients entering the investigation programme, 9 completed the protocol. Of the 3 who did not, 2 were withdrawn after a 3-week period of drug administration; I developed left ventricular failure which settled on digoxin and diuretic therapy. The other experienced an episode of severe ischaemic cardiac pain associated with marked $T$ wave inversion in the praecordial leads of the electrocardiogram; these changes, which were not associated with an increase in serum myocardial enzymes, regressed completely, but the patient was not given a further course of treatment with amiodarone. The third patient failed to attend the clinic at the appropriate time, but data were available after 3 weeks of amiodarone therapy. Of the I2 patients studied, 8 had complained of effort angina before entry into the trial. Five of the 8 either became free of pain or were significantly improved when they were given amiodarone. The remainder had mild symptoms only and derived no benefit from the drug.

\section{Thyroid function}

None of the patients had clinical or laboratory evidence of thyroid disease before amiodarone was started. All patients given amiodarone remained clinically euthyroid during the entire period of drug administration. Mean half relaxation time of the ankle jerks before treatment was $246 \pm 44$ ( \pm SD) $\mathrm{ms}$ and at the end of treatment, $237 \pm 46( \pm S D) \mathrm{ms}$. The mean serum cholesterol level at the beginning of therapy was $6.6 \pm 0.5 \mathrm{mmol} / 1(255 \pm 18( \pm S D)$ $\mathrm{mg} / 100 \mathrm{ml}$ ) and at the time therapy was completed the level was $7.0 \pm 0.8 \mathrm{mmol} / 1(269 \pm 33( \pm S D)$ $\mathrm{mg} / \mathrm{ro0} \mathrm{ml}$ ). Analysis of paired samples showed no significant difference in either of these indices of possible hypothyroidism.

After 3 weeks of treatment with amiodarone, serum PBI in all patients rose to immeasurably high levels. The increase was attributable to the iodine content of amiodarone and provided confirmatory evidence that the patients were taking the tablets. Good laboratory evidence against the development of primary hypothyroidism (Evered et al., 1973) was obtained by the fact that serum TSH remained within normal limits (i.e. less than $4 \mu \mathrm{U} / \mathrm{ml}$ ) in all patients but one. This patient showed no other evidence of hypothyroidism and though his serum TSH level at the beginning of the study was $I I .5 \mu \mathrm{U} / \mathrm{ml}$, it subsequently fell to normal levels during the remaining period of observation. Furthermore, there was no significant change in $T_{3}$ resin uptake suggesting no

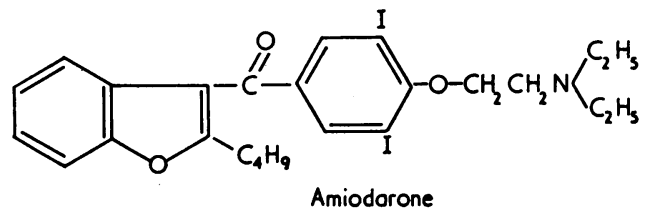

FIG. I Structural formula of amiodarone. The compound has 37.2 per cent by weight of iodine.

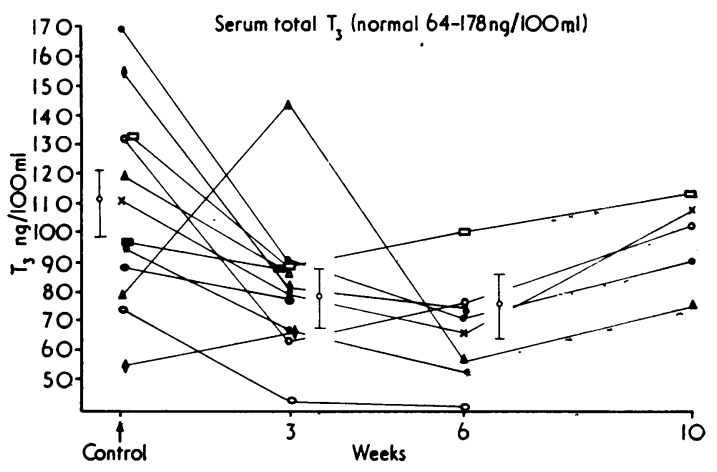

FIG. 2 The effect of amiodarone on total serum tri-iodothyronine in patients with ischaemic heart disease.

Each symbol shown represents a value from a different patient. The mean $\pm S E M$ 's shown are for the whole group. The values of $T_{3}$ at 3 and 6 weeks after amiodarone are significantly different $(P<0.01)$ from the control value. Except in one patient, the actual values have not deviated outside the normal range. 


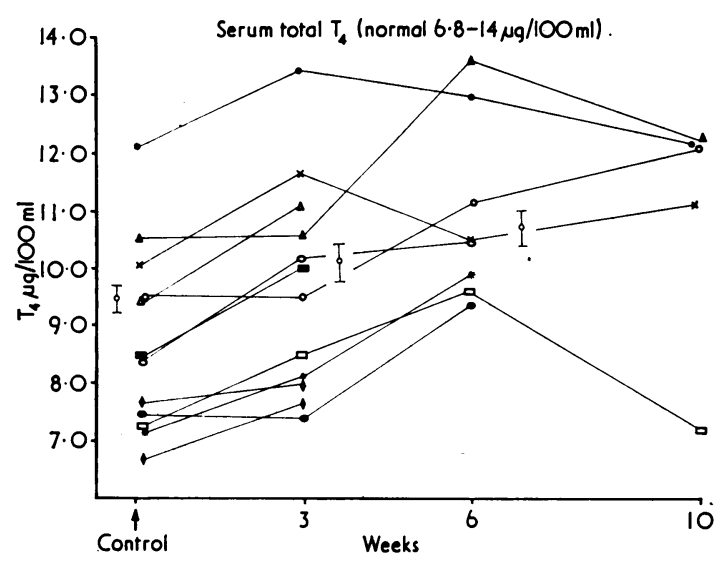

FIG. 3 The effect of amiodarone on total serum thyroxine in patients with ischaemic heart disease.

Each symbol on the diagram represents a value from a different patient. The mean $\pm S E M$ 's are shown for the whole group. The mean $T_{4}$ level after 6 weeks of treatment was significantly different $(P<0.01)$ from the control value. The actual values have not, however, deviated outside the normal range.

alteration in the levels of thyroxine-binding proteins.

The changes in the serum levels of $T_{3}$ and $T_{4}$ during amiodarone therapy are shown in Fig. 2 and 3, respectively. After 3 weeks of treatment with the

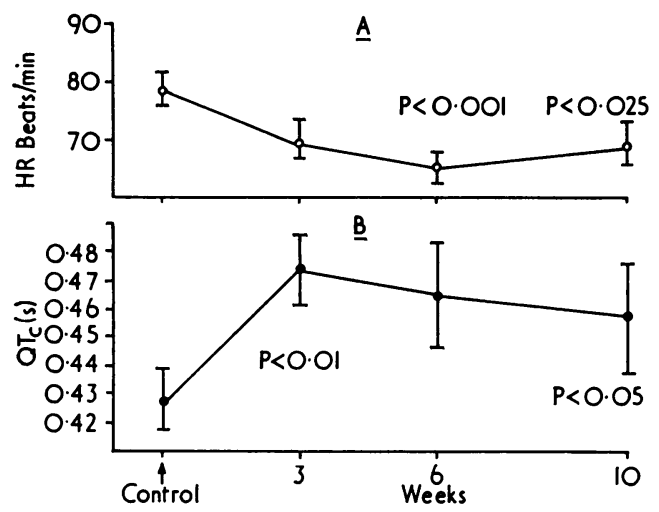

FIG. 4 Changes in heart rate and the $Q T c$ interval of the electrocardiogram during amiodarone administration. A) There is progressive reduction in heart rate after amiodarone was started with the development of significant bradycardia at 6 weeks. Recovery is not complete 4 weeks after stopping the drug. $B$ ) the change in QTc interval is maximal at 3 weeks after amiodarone was started. The $Q T c$ is still significantly prolonged 4 weeks after stopping the drug. drug, $T_{3}$ levels fell significantly $(P<0.0 \mathrm{I})$ and remained low $(P<0.01)$ after 6 weeks of therapy. After cessation of amiodarone intake, the levels of $\mathrm{T}_{3}$ tended to rise again but recovery was not complete 4 weeks later. Changes were not monitored thereafter. It is noteworthy, however, that though serum $T_{3}$ levels fell significantly during the administration of amiodarone, the actual values of the hormone in the serum usually remained within the normal range for the laboratory. An opposite trend in the serum $\mathrm{T}_{4}$ levels during amiodarone administration was found. Serum $T_{4}$ increased in all patients who took the drug for a period greater than 3 weeks (Fig. 3) but, as in the case of $T_{3}$ levels, the absolute levels remained within the laboratory range of normal values. The differing directional change in $T_{4}$ and $T_{3}$ levels during amiodarone therapy is reflected in the pronounced alteration in the $T_{4} / T_{3}$ ratio. Before amiodarone was given, this mean ratio was $8 I \pm 8(n=12)$; 6 weeks after amiodarone treatment it rose to $179 \pm 7 \quad(n=8)$. The change was highly significant $(P<0.00 \mathrm{I})$.

\section{Cardiovascular effects}

The changes in heart rate and the QTc interval on the electrocardiogram during amiodarone administration are presented in Fig. 4A and B. Three weeks after amiodarone was started, the mean reduction in heart rate for the group was about Io per cent; at 6 weeks, it was about 15 per cent. When the drug was withdrawn, heart rate tended to increase, but recovery was not complete 4 weeks later. There were no significant changes in systolic or diastolic blood pressures throughout the entire period of study.

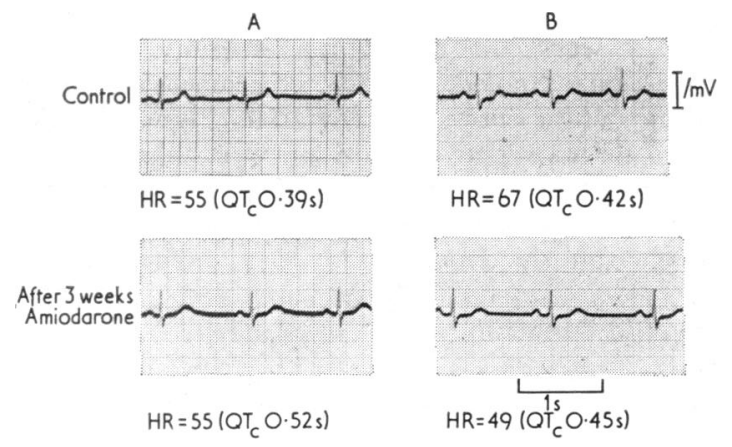

FIG. 5 Effects of amiodarone on QTc interval of the electrocardiogram in two patients. In patient $A$, the $Q T c$ interval was lengthened without a change in heart rate; in patient $B$, bradycardia as well as QTC prolongation developed 3 weeks after amiodarone was started. 
For this reason, these results are not presented in detail.

The alterations in QTc interval during drug treatment are presented in Fig. 4B. During amiodarone therapy a significant prolongation in the QTc interval was demonstrated in all patients, the maximal effect occurring 3 weeks after the start of the drug. Fig. 5 illustrates the change in 2 patients. It will be seen that in patient $A$ whose control heart rate was low ( $55 / \mathrm{min})$, prolongation in the QTc interval occurred without a further reduction in rate. In patient $B$, bradycardia as well as lengthening of the QTc interval were produced by amiodarone. These changes persisted during continued administration of amiodarone, and significant prolongation of the QTc interval was still evident 4 weeks after stopping the drug. There were no changes in the QRS duration or the PR interval during the period of drug therapy.

\section{Discussion}

The data presented here confirm previous observations (Facquet et al., 1970) that chronic administration of amiodarone in patients with cardiac disease leads to the development of a moderate degree of bradycardia of gradual onset associated with a significant degree of prolongation of the QTc interval of the electrocardiogram. Our results provide further and reasonably decisive evidence that these changes, which were found to regress slowly on withdrawal of treatment, are not caused by impaired thyroid function. However, it was of interest to find that the drug produced a significant increase in the levels of $T_{4}$ in association with a comparable decline in serum $T_{3}$ levels. The reason for these contrasting effects is not clear but may be related to the iodine load produced by amiodarone which has 37.2 per cent iodide by weight. Under these circumstances, it may be speculated that a preferential synthesis of $T_{4}$ in the thyroid gland occurs at the expense of $T_{3}$. On the other hand, the possibility that an effect on $\mathrm{T}_{4}$ metabolism by an inhibition of peripheral breakdown of $T_{4}$ to $T_{3}$ (Larsen, 1972), which may also account for the observed changes in the levels of these hormones during therapy, cannot be excluded.

The efficacy of amiodarone as an antianginal agent is now well established (Vastesaeger et al., 1967; Barzin and Fréson, 1969). In chronic animal studies (Charlier et al., I968) the drug was found to affect the major determinants of myocardial oxygen consumption. The development of significant bradycardia in our own studies in anginal patients would also imply a reduction in myocardial oxygen utilization contributing to the drug's antianginal efficacy. Barzin and Fréson (1969) found that amiodarone produced a fall in the values of the rate-systolic pressure product which has been used as an index of myocardial oxygen utilization (Robinson, 1967). Our own small study was not designed to evaluate the antianginal efficacy of amiodarone, but it is nevertheless of interest that an improvement in symptoms was found in the majority of our patients. The occurrence of severe chest pain with ischaemic changes in the electrocardiogram occurring during amiodarone therapy in one patient seems fortuitous. It has not been reported in other studies in which amiodarone was given to large numbers of patients for prolonged periods of time (Vastesaeger et al., 1967). Similarly, the development of left ventricular failure in another patient may not have been related to drug treatment. Amiodarone is unlikely to alter the adrenergic drive to the heart since it produces a weak non-cempetitive sympathetic antagonism, with little effect on left ventricular function (Charlier et al., 1968; Charlier, 1970). Barzin and Fréson (1969) have shown that cardiac output after I month's treatment with a daily dose of $600 \mathrm{mg}$ amiodarone was not altered significantly, even in those patients who had cardiac decompensation.

The electrocardiographic changes during amiodarone therapy found in our study are of particular significance in relation to the mechanism of the drug's known antiarrhythmic actions in animals (Singh and Vaughan Williams, 1970) and in man (Van Schepdael and Solvay, 1970). The prolongation in the QTc in the absence of any changes in the PR interval or the QRS duration extends the observations of Facquet et al. (1970). The findings are in line with the results of our electrophysiological investigations in animals (Singh and Vaughan Williams, 1970) and with those of Olsson, Brorson, and Varnauskas (1973) in man. Using suction electrodes, these authors showed that chronic administration of amiodarone in patients led to a prolongation in the repolarization phase of the monophasic right atrial action potential. These results are of special interest in relation to the finding that patients with a tendency to relapse to atrial fibrillation after DC conversion to sinus rhythm had more rapid atrial repolarization than those who maintained sinus rhythm (Olsson, Cotoi, and Varnauskas, 1971). Accelerated repolarization of the atrial action potential has also been reported in experimental thyrotoxicosis, and the converse in hypothyroidism (Freedberg et al., 1970). The effects of amiodarone on atrial fibrillation in thyrotoxicosis and on the relapse rate after cardioversion of this arrhythmia in other conditions may thus be of interest.

A feature of interest suggested by the results of 
the present investigations when considered in relation to those of our previous study (Singh and Vaughan Williams, I970) is the fact that it might be possible to block selectively the action of thyroxine on the heart without interfering with the effects of the hormone on other tissues. For example, in the present studies amiodarone had no significant effects on peripheral tissues as suggested by clinical features and the maintenance of normal ankle jerk half relaxation time at a time when the drug delayed repolarization and produced bradycardia. In animals, Singh and Vaughan Williams (1970) showed that simultaneous administration of amiodarone and thyroxine prevented the prolongation of the cardiac action potential. This effect was shown not to be caused by iodide in the drug since an equivalent dose of iodide given as potassium iodide produced no change in the action potential. The precise mechanism whereby amiodarone produces its cardiac effects remains uncertain. It is possible, however, that the drug either selectively blocks the effect of endogenous thyroxine on the heart, or, as suggested elsewhere (Singh and Vaughan Williams, 1970), the changes produced are secondary to a reduction by amiodarone of cardiac metabolism in parallel, as it were, with thyroxine-dependent pathways.

We would like to express our thanks to Drs. R. M. Norris and C. J. Mercer for allowing us to study patients under their care. We are also indebted to Mr. T. Lim who performed the radioimmunoassay for TSH and Miss J. Jacobson who carried out the radioimmunoassay for the total $T_{3}$. The investigations were in part supported by the Medical Research Council of New Zealand.

\section{References}

Barzin, J., and Fréson, A. (1969). Essais cliniques de l'aminodarone dans les affections coronariennes. Bruxellesmédicale, 49, 105.

Bazett, H. C. (1920). An analysis of the time-relations of electrocardiograms. Heart, 7, 353.
Charlier, R. (1970). Cardiac actions in the dog of a new antagonist of adrenergic excitation which does not produce competitive blockade of adrenoceptors. British fournal of Pharmacology, 39, 668.

Charlier, R., Deltour, G., Baudine, A., and Chaillet, F. (1968). Pharmacology of amiodarone: an anti-anginal drug with a new biological profile. Arzneimittel-Forschung, 18, 1408.

Evered, D. C., Ormston, B. J., Smith, P. A., Hall, R., and Bird, T. (1973). Grades of hypothyroidism. British Medical fournal, $\mathbf{1}, 657$.

Facquet, J., Nivet, M., Grosgogeat, Y., Alhomme, P., and Vachon, J. (1970). L'influence de l'aminodarone sur le rhythme cardiaque et l'électrocardiogramme. Thérapie, 25, 335.

Freedberg, A. S., Papp, J. Gy., and Vaughan Williams, E. M. (1970). The effect of altered thyroid state on atrial intracellular potentials. Fournal of Physiology, 207, 357.

Harrison, M. T., and Cameron, A. J. V. (1965). Iodine-induced hypothyroidism due to benziodarone. British Medical fournal, $1,840$.

Larsen, P. R. (1972). Triiodothyronine: review of recent studies of its physiology and pathophysiology in man. Metabolism, 21, 1073.

Olsson, S. B., Brorson, L., and Varnauskas, E. (1973). Class 3 antiarrhythmic action in man. Observations from monophasic action potential recordings and amiodarone treatment. British Heart fournal, 35, 1255.

Olsson, S. B., Cotoi, S., and Varnauskas, E. (1971). Monophasic action potential and sinus rhythm stability after conversion of atrial fibrillation. Acta Medica Scandinavica, 190, 381.

Robinson, B. F. (1967). Relation of heart rate and systolic blood pressure to the onset of pain in angina pectoris. Circulation, 35, 1073.

Rosenbaum, M. B., Chale, P. A., Ryba, D., and Elizari, M. V. (1974). Control of tachyarrhythmias associated with Wolff-Parkinson-White syndrome by amiodarone hydrochloride. American fournal of Cardiology, 34, 215.

Singh, B. N., and Vaughan Williams, E. M. (I970). The effect of amiodarone, a new anti-anginal drug, on cardiac muscle. British fournal of Pharmacology, 39, 657.

Van Schepdael, J., and Solvay, H. (I970). Etude clinique de l'amiodarone dans les troubles du rhythme cardiaque. Presse Médicale, 78, 1849.

Vastesaeger, M., Gillot, P., and Rasson, G. (1967). Etude clinique d'une nouvelle médication anti-angoreuse. Acta Cardiologica, 22, 483.

Requests for reprints to Dr. B. N. Singh, Department of Medicine, Auckland University School of Medicine, Auckland, New Zealand. 